PHYSICAL REVIEW D 91, 074028 (2015)

\title{
LHC constraints on gauge boson couplings to dark matter
}

\author{
Andreas Crivellin, ${ }^{1, *}$ Ulrich Haisch, ${ }^{2,1, \dagger}$ and Anthony Hibbs ${ }^{2, *}$ \\ ${ }^{1}$ CERN Theory Division, CH-1211 Geneva 23, Switzerland \\ ${ }^{2}$ Rudolf Peierls Centre for Theoretical Physics, University of Oxford, Oxford OX1 3PN, United Kingdom
}

(Received 20 January 2015; published 28 April 2015)

\begin{abstract}
Collider searches for energetic particles recoiling against missing transverse energy $\left(E_{T}\right)$ allow us to place strong bounds on the interactions between dark matter (DM) and standard model (SM) particles. In this article we update and extend LHC constraints on effective dimension-7 operators involving DM and electroweak gauge bosons. A concise comparison of the sensitivity of the monophoton, mono- $W$, mono- $Z$, mono- $W / Z$, and invisible Higgs-boson decays in the vector boson fusion mode and the monojet channel is presented. Depending on the parameter choices, either the monophoton or the monojet data provide the most stringent bounds at the moment. We furthermore explore the potential of improving the current $8 \mathrm{TeV}$ limits at $14 \mathrm{TeV}$. Future strategies capable of disentangling the effects of the different effective operators involving electroweak gauge bosons are discussed as well.
\end{abstract}

DOI: $10.1103 /$ PhysRevD.91.074028

PACS numbers: 13.85.Rm, 95.30.Cq, 95.35.+d

\section{INTRODUCTION}

Because of their potential connection to dark matter (DM), searches for $E_{T}$ represent one of the main lines of LHC research. These searches can be categorized based on the type of Standard Model (SM) particles that recoil against DM. By now, ATLAS and CMS have considered a plethora of different final states in DM searches containing jets of hadrons, gauge bosons, heavy quarks and even the Higgs boson (see e.g. [1] for a recent review of the experimental status).

In most cases these studies are performed in the context of an effective field theory (EFT) which correctly captures the physics of heavy particles mediating the interactions between DM and SM fields, if the mediators are heavy enough to be integrated out. Below we will consider the effective Lagrangian

$$
\mathcal{L}_{\text {eff }}=\sum_{k=B, W, \tilde{B}, \tilde{W}} \frac{C_{k}(\mu)}{\Lambda^{3}} O_{k}
$$

which contains the following four $S U(2)_{L} \times U(1)_{Y}$ gaugeinvariant dimension-7 operators

$$
\begin{array}{rlrl}
O_{B} & =\bar{\chi} \chi B_{\mu \nu} B^{\mu \nu}, & & O_{W}=\bar{\chi} \chi W_{\mu \nu}^{i} W^{i, \mu \nu}, \\
O_{\tilde{B}}=\bar{\chi} \chi B_{\mu \nu} \tilde{B}^{\mu \nu}, & & O_{\tilde{W}}=\bar{\chi} \chi W_{\mu \nu}^{i} \tilde{W}^{i, \mu \nu} .
\end{array}
$$

*andreas.crivellin@cern.ch

u.haisch1@physics.ox.ac.uk

‡Anthony.Hibbs@physics.ox.ac.uk

Published by the American Physical Society under the terms of the Creative Commons Attribution 3.0 License. Further distribution of this work must maintain attribution to the author(s) and the published article's title, journal citation, and DOI.
Here $\Lambda$ represents the scale of new physics at which the higher-dimensional operators (1) are generated, i.e. the scale where the messenger particles are removed as active degrees of freedom. The DM particle $\chi$ can be both a Dirac or a Majorana fermion and $B_{\mu \nu}=\partial_{\mu} B_{\nu}-\partial_{\nu} B_{\mu}$ $\left[W_{\mu \nu}^{i}=\partial_{\mu} W_{\nu}^{i}-\partial_{\nu} W_{\mu}^{i}+g_{2} \epsilon^{i j k} W_{\mu}^{j} W_{\mu}^{k}\right]$ is the $U(1)_{Y}$ $\left[S U(2)_{L}\right]$ field strength tensor, while $\tilde{B}_{\mu \nu}=1 / 2 \epsilon_{\mu \nu \lambda \rho} B^{\lambda \rho}$ $\left[\tilde{W}_{\mu \nu}^{i}=1 / 2 \epsilon_{\mu \nu \lambda \rho} W^{i, \lambda \rho}\right]$ denotes its dual and $g_{2}$ is the weak coupling constant.

The operators introduced in (2) appear in models of Rayleigh DM (see for instance [2-4]). They are special in the sense that, up to dimension 7 , they are the only effective interactions which lead to velocity-suppressed annihilation rates of DM to photon pairs [5-7]. While the sensitivity of future direct detection experiments may allow us to set novel bounds on the Wilson coefficients $C_{B}(\Lambda)$ and $C_{W}(\Lambda)$ for heavy DM particles with $m_{\chi} \gtrsim 1 \mathrm{TeV}$ once loop effects are taken into account [8], in the case of light DM the leading [and for $C_{\tilde{B}}(\Lambda)$ and $C_{\tilde{W}}(\Lambda)$ the only] restrictions arise and will continue to arise from collider searches involving large amounts of $E_{T}$. In fact, the DM-SM interactions (2) have been constrained using $7 \mathrm{TeV}$ and $8 \mathrm{TeV}$ LHC data on invisible decays of the Higgs boson in the vector boson fusion (VBF) mode [9] as well as the $E_{T}+Z[10,11]$, monophoton [12] and $E_{T}+W$ [13] channels.

The main goal of this article is twofold. First, to update the existing constraints by taking into account the latest monophoton [14,15], $E_{T}+W / Z(\rightarrow$ hadrons) [16] and VBF $h \rightarrow$ invisible [17] searches. Second, to extend the studies [9-13] by considering in addition the $E_{T}+W(\rightarrow$ leptons $)$ channel $[18,19]$ as well as the newest monojet data [20]. An assortment of Feynman diagrams that lead to the $E_{T}$ signatures investigated in the following are displayed in Fig. 1.

Our analysis shows that depending on the choice of parameters, either the monophoton or the monojet data give 

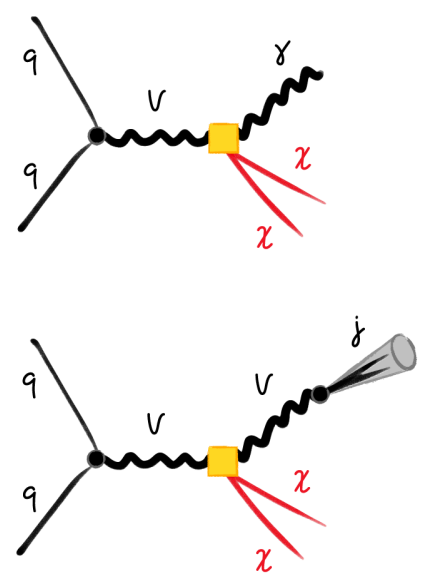
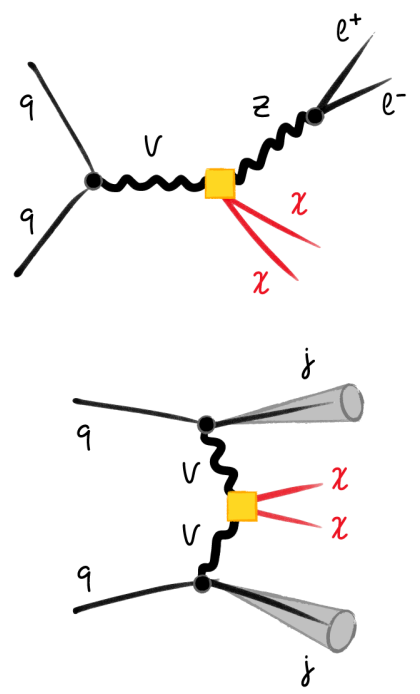

FIG. 1 (color online). Representative examples of graphs that generate a $E_{T}+\gamma, \quad E_{T}+Z\left(\rightarrow \ell^{+} \ell^{-}\right), \quad E_{T}+W / Z(\rightarrow j)$ or $E_{T}+2 j$ signal. The operator insertions are indicated by yellow squares while SM vertices are represented by black dots. Propagators labeled by $V$ include all possible photon, $Z$-boson or $W$-boson exchanges. See text for further details.

rise to the strongest restrictions at present. By combining the information on all available channels we are thus able to derive bounds on the coefficients $C_{k}(\Lambda) / \Lambda^{3}$ in (1) that improve on the existing limits. Building upon [21], we furthermore demonstrate that measurements of the jet-jet azimuthal angle difference in $E_{T}+2 j$ events may be used to disentangle whether the DM bilinear $\bar{\chi} \chi$ couples more strongly to the combination $B_{\mu \nu} B^{\mu \nu}\left[W_{\mu \nu}^{i} W^{i, \mu \nu}\right]$ or the product $B_{\mu \nu} \tilde{B}^{\mu \nu}\left[W_{\mu \nu}^{i} \tilde{W}^{i, \mu \nu}\right]$ of field strength tensors. Similar ideas have also been brought forward in [9].

The outline of this article is as follows. In Sec. II we review the existing LHC searches for $E_{T}$ signatures that we will use to constrain the effective interactions (2). In Sec. III we derive the restrictions on the parameter space by combining all individual search modes, commenting also on how future measurements may improve these limits. This section contains in addition a discussion of the azimuthal angle correlations between the two jets in the $E_{T}+2 j$ channel. Our conclusions are presented in Sec. IV.

\section{SEARCH CHANNELS}

In this section we list the various cuts and the values of the fiducial cross section $\left(\sigma_{\text {fid }}\right)$ of each individual $E_{T}$ channel. This information will be used in the next section to set limits on the coefficients $C_{k}(\Lambda) / \Lambda^{3}$ appearing in the effective Lagrangian (1).

\section{A. Monophoton signal}

We begin with the monophoton signal, which has recently been searched for by both CMS [14] and ATLAS [15].
Since the former search leads to the stronger restrictions, we employ the CMS results, which are based on $19.6 \mathrm{fb}^{-1}$ of $8 \mathrm{TeV}$ data. The relevant cuts are

$$
E_{T}>140 \mathrm{GeV}, \quad\left|\eta_{\gamma}\right|<1.4442,
$$

where $\eta_{\gamma}$ denotes the pseudorapidity of the photon. The CMS Collaboration performs the measurement in six different signal regions with a varying cut on the transverse momentum of the photon $\left(p_{T, \gamma}\right)$. Note that due to the higherdimensional nature of the operators (2), the $E_{T}+\gamma$ signal has a rather hard $p_{T, \gamma}$ spectrum. As a result, we find that the most severe cut of $p_{T, \gamma}>700 \mathrm{GeV}$ gives the strongest bounds on the parameter space in our case. The corresponding 95\% confidence level (C.L.) limit on the fiducial cross section reads

$$
\sigma_{\text {fid }}\left(p p \rightarrow E_{T}+\gamma\right)<0.22 \mathrm{fb} .
$$

\section{B. Mono- $Z$ signal}

In the case of the $E_{T}+Z\left(\rightarrow \ell^{+} \ell^{-}\right)$search channel, we use the ATLAS results [11] that utilize $20.3 \mathrm{fb}^{-1}$ of $8 \mathrm{TeV}$ data. The selection criteria relevant to our analysis are

$p_{T, \ell}>20 \mathrm{GeV}, \quad\left|\eta_{\ell}\right|<2.5, \quad m_{\ell \ell} \in[76,106] \mathrm{GeV}$,

$\left|\eta_{\ell \ell}\right|<2.5, \quad \frac{\left|p_{T, \ell \ell}-E_{T}\right|}{p_{T, \ell \ell}}<0.5$.

Here $m_{\ell \ell}, \eta_{\ell \ell}$ and $p_{T, \ell \ell}$ denote the invariant mass, the pseudorapidity and the transverse momentum of the dilepton system, respectively. The ATLAS analysis defines four signal regions with different lower $E_{T}$ thresholds. As it turns out, in the considered case the requirement $E_{T}>350 \mathrm{GeV}$ gives rise to the best bounds. Including $Z$-boson decays to both electrons and muons $(\ell=e, \mu)$, the ATLAS experiment obtains for this $E_{T}$ cut the following 95\% C.L. bound

$$
\sigma_{\text {fid }}\left(p p \rightarrow E_{T}+Z\left(\rightarrow \ell^{+} \ell^{-}\right)\right)<0.27 \mathrm{fb} .
$$

\section{Mono- $W$ signal}

Both ATLAS [18] and CMS [19] have searched for a mono- $W$ signal in the leptonic decay mode. We find that the ATLAS search for the $\mu \nu_{\mu}$ final state, which uses $20.3 \mathrm{fb}^{-1}$ of $8 \mathrm{TeV}$ data, gives the strongest constraints, and thus we consider only this channel. The most important experimental cuts are

$$
\begin{aligned}
p_{T, \mu} & >45 \mathrm{GeV}, \quad\left|\eta_{\mu}\right| \in[0,1] \cup[1.3,2], \\
m_{T} & =\sqrt{2 p_{T, \mu} E_{T}\left(1-\cos \varphi_{\mu E_{T}}\right)},
\end{aligned}
$$

where $m_{T}$ is the transverse mass which depends on the angle $\varphi_{\mu E_{T}}$ between the $p_{T, \mu}$ and the $E_{T}$ vectors. ATLAS 
sets bounds on $\sigma_{\text {fid }}$ for three different $m_{T}$ cuts, and like in the case of the monophoton signal, we observe that the strongest restriction of $m_{T}>843 \mathrm{GeV}$ provides the best limits on the interactions (2). At 95\% C.L. the bound on the corresponding fiducial signal cross section is given by

$$
\sigma_{\text {fid }}\left(p p \rightarrow E_{T}+W\left(\rightarrow \mu \nu_{\mu}\right)\right)<0.54 \mathrm{fb} .
$$

\section{Mono- $W / Z$ signal}

The ATLAS search [16] looks for a $E_{T}+W / Z$ signal, where the $W$ or $Z$ boson decays hadronically. This analysis is based on $20.3 \mathrm{fb}^{-1}$ of $8 \mathrm{TeV}$ data; jet candidates are reconstructed using the Cambridge/Aachen (C/A) algorithm [22] with a radius parameter $R=1.2$ and subjected to a mass-drop filtering procedure [23]. Events are required to have at least one C/A jet with

$$
\begin{aligned}
p_{T, j} & >250 \mathrm{GeV}, \quad\left|\eta_{j}\right|<1.2, \\
m_{j} & \in[50,120] \mathrm{GeV}, \quad \sqrt{y}>0.4 .
\end{aligned}
$$

Here $m_{j}$ refers to the mass of the large-radius jet, while $\sqrt{y}=\min \left(p_{T, j_{1}}, p_{T, j_{2}}\right) \sqrt{\left(\Delta \phi_{j_{1} j_{2}}\right)^{2}+\left(\Delta \eta_{j_{1} j_{2}}\right)^{2}} / m_{j}$ is a measure of the momentum balance of the two leading subjets $j_{1}$ and $j_{2}$ contained in the C/A jet. The 95\% C.L. limits on the fiducial cross section depend also on the imposed $E_{T}$ threshold, and it turns out that the stronger of the two cuts, i.e. $E_{T}>500 \mathrm{GeV}$, provides the most stringent constraints. In this case, the relevant limit on the fiducial cross section is

$$
\sigma_{\text {fid }}\left(p p \rightarrow E_{T}+W / Z(\rightarrow \text { hadrons })\right)<2.2 \mathrm{fb} .
$$

\section{E. Monojet signal}

One can also use monojet events to constrain the operators in (2), since the corresponding searches allow for the presence of a secondary jet. Here we will employ the newest CMS results [20], which make use of $19.7 \mathrm{fb}^{-1}$ of $8 \mathrm{TeV}$ data. Like CMS, we reconstruct jets using an anti- $k_{t}$ algorithm [24] with radius parameter $R=0.5$. The relevant selection cuts are

$$
\begin{aligned}
p_{T, j_{1}} & >110 \mathrm{GeV}, & & \left|\eta_{j_{1}}\right|<2.4, \\
p_{T, j_{2}} & >30 \mathrm{GeV}, & & \left|\eta_{j_{2}}\right|<4.5, \\
\Delta \phi_{j_{1} j_{2}} & <2.5, & &
\end{aligned}
$$

where $\Delta \phi_{j_{1} j_{2}}$ is the azimuthal separation of the two leading jets. Another important selection criterion is the imposed jet veto [25], which rejects events if they contain a tertiary jet with $p_{T, j_{3}}>30 \mathrm{GeV}$ and $\left|\eta_{j_{3}}\right|<4.5$. The CMS measurement is performed for seven different $E_{T}$ regions, and we find that for the considered interactions the highest sensitivity is obtained for $E_{T}>500 \mathrm{GeV}$. The corresponding 95\% C.L. limit on the fiducial cross section reads

$$
\sigma_{\text {fid }}\left(p p \rightarrow E_{T}+2 j\right)<6.1 \mathrm{fb} \text {. }
$$

\section{F. VBF invisible Higgs-boson decays}

Last but not least, we consider the results of the CMS search for invisible decays of the Higgs boson in the VBF channel [17], which uses an $8 \mathrm{TeV}$ data sample, corresponding to an integrated luminosity of $19.5 \mathrm{fb}^{-1}$. Jets are reconstructed employing an anti- $k_{t}$ clustering algorithm with $R=0.5$, and subject to the following requirements

$$
\begin{aligned}
p_{T, j_{1}}, p_{T, j_{2}} & >50 \mathrm{GeV}, \quad\left|\eta_{j_{1}}\right|,\left|\eta_{j_{2}}\right|<4.7, \\
\eta_{j_{1}} \cdot \eta_{j_{2}} & <0, \quad \Delta \eta_{j_{1} j_{2}}>4.2, \\
m_{j_{1} j_{2}} & >1100 \mathrm{GeV}, \quad \Delta \phi_{j_{1} j_{2}}<1.0 .
\end{aligned}
$$

The missing-energy cut is $E_{T}>130 \mathrm{GeV}$ and a central jet veto is imposed to any event that has a third jet with $p_{T, j_{3}}>$ $30 \mathrm{GeV}$ and a pseudorapidity between those of the two tagging jets. For these cuts, CMS obtains the following 95\% C.L. bound on the fiducial cross section

$$
\sigma_{\mathrm{fid}}\left(p p \rightarrow E_{T}+2 j\right)<6.5 \mathrm{fb} .
$$

\section{NUMERICAL RESULTS}

In order to determine the cross section for the $E_{T}$ signals associated to the effective operators (2), we have implemented each of them in FeynRules [26], generating a universal FeynRules output (UFO) [27]. The actual event generation has been performed at leading order with MadGraph 5 [28] utilizing CTEQ6L1 parton distributions [29]. Parton-shower effects and hadronization corrections have been included by means of PYTHIA 8 [30] and jets constructed using FastJet 3 [31]. We employ Delphes 3 [32] as a fast detector simulation to estimate the reconstruction efficiencies for the different $E_{T}$ signals. The efficiencies that we find amount to around $70 \%$ for the monophoton signal, $60 \%$ in both the mono- $Z$ and mono- $W$ case and $65 \%$ for the mono- $W / Z$ signature. These findings agree with [15] for the $E_{T}+\gamma$, [11] for the $E_{T}+Z$, [13] for the $E_{T}+W$ and [16] for the $E_{T}+W / Z$ signal. For the monojet signal and the search for invisible decays of the Higgs boson in the VBF channel, we find reconstruction efficiencies in the ballpark of $95 \%$.

Our Monte Carlo (MC) implementation has been validated by reproducing the numerical results of $[11,12]$ within theoretical uncertainties. These errors have been assessed by studying the scale ambiguities of our results. We have used the default dynamical scale choice of MadGraph 5, varying the scale factor in the range $[1 / 2,2]$. We find that the predictions for the monophoton, $E_{T}+Z\left(\rightarrow \ell^{+} \ell^{-}\right)$and $E_{T}+W\left(\rightarrow \mu \nu_{\mu}\right)$ cross sections calculated in this way vary by around $\pm 15 \%$, while in the case of the $E_{T}+W / Z(\rightarrow$ hadrons $)$, the monojet and the VBF $h \rightarrow$ invisible signal, relative differences of about $\pm 20 \%$ are obtained. Note that these errors are smaller than 
those found in [25,33-35], since all the tree-level $E_{T}$ cross sections considered in our work do not explicitly depend on $\alpha_{s}$. The quoted uncertainties thus reflect only the ambiguities related to the change of factorization scale, but not renormalization scale.

\section{A. Dependence on a single Wilson coefficient}

In Fig. 2 we present the limits on the new-physics scale $\Lambda$ for $C_{\tilde{B}}(\Lambda)=C_{\tilde{W}}(\Lambda)=0$ and the two choices $C_{B}(\Lambda)=1$, $C_{W}(\Lambda)=0$ (upper panel) and $C_{B}(\Lambda)=0, C_{W}(\Lambda)=1$ (lower panel) for the Wilson coefficients evaluated at $\Lambda$. The shown predictions correspond to Dirac DM and the widths of the colored bands illustrate the impact of scale variations. For $C_{B}(\Lambda)=1, C_{W}(\Lambda)=0$, one observes that
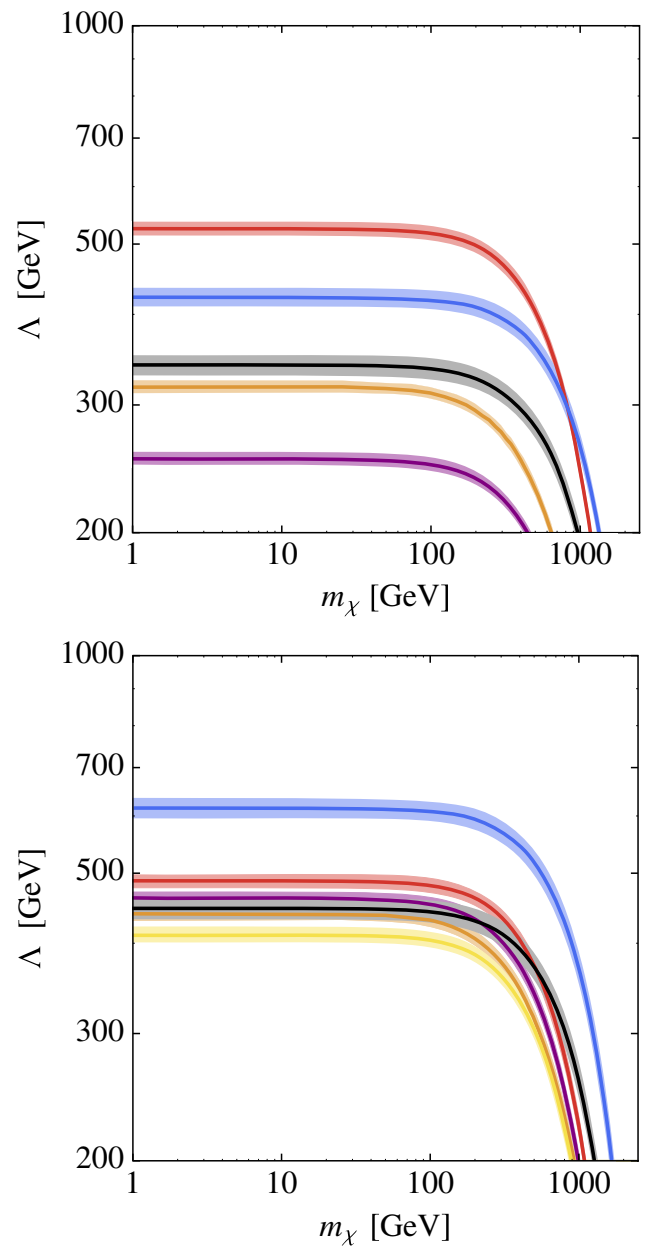

FIG. 2 (color online). Assortment of LHC bounds on the newphysics scale $\Lambda$, assuming $C_{B}(\Lambda)=1, C_{W}(\Lambda)=0$ (upper panel) and $C_{B}(\Lambda)=0, C_{W}(\Lambda)=1$ (lower panel). In both cases the DM particles are taken to be Dirac and $C_{\tilde{B}}(\Lambda)=C_{\tilde{W}}(\Lambda)=0$. The colored curves correspond to the limits arising from the latest monophoton (red), $E_{T}+Z\left(\rightarrow \ell^{+} \ell^{-}\right)$(orange), $E_{T}+W\left(\rightarrow \mu \nu_{\mu}\right)$ (yellow), $E_{T}+W / Z(\rightarrow$ hadrons) (purple), monojet (blue) and VBF $h \rightarrow$ invisible (grey) searches. The width of the bands reflect the associated scale uncertainties. the monophoton search [14] provides the strongest constraints in most of the parameter space. Numerically, we find that the scale $\Lambda$ has to satisfy $\Lambda \gtrsim 510 \mathrm{GeV}$ for $m_{\chi} \lesssim$ $100 \mathrm{GeV}$ in order to meet the $95 \%$ C.L. requirement (4). In the case $C_{B}(\Lambda)=0, C_{W}(\Lambda)=1$, on the other hand, the latest monojet data [20] impose the leading restrictions. At 95\% C.L., the inequality (12) translates into a lower limit of $\Lambda \gtrsim 600 \mathrm{GeV}$ for $\mathrm{DM}$ masses below $100 \mathrm{GeV}$. The shown limits also hold in the case that $C_{\tilde{B}}(\Lambda)=1, C_{\tilde{W}}(\Lambda)=0$ or $C_{\tilde{B}}(\Lambda)=0, \quad C_{\tilde{W}}(\Lambda)=1$ and $C_{B}(\Lambda)=C_{W}(\Lambda)=0$, while for Majorana DM the constraints on $\Lambda$ would be stronger by around $12 \%$. Note finally that $E_{T}+W\left(\rightarrow \mu \nu_{\mu}\right)$ searches do not provide any constraint on scenarios with $C_{W}(\Lambda)=C_{\tilde{W}}(\Lambda)=0$.

To better understand the restrictions imposed by the various search channels, we consider the Feynman rules associated to the effective operators $O_{B}$ and $O_{W}$ entering (1). In momentum space, the resulting interactions between pairs of DM particles and SM gauge bosons take the form

$$
\frac{4 i}{\Lambda^{3}} g_{V_{1} V_{2}}\left(p_{1}^{\mu_{2}} p_{2}^{\mu_{1}}-g^{\mu_{1} \mu_{2}} p_{1} \cdot p_{2}\right),
$$

where $p_{i}\left(\mu_{i}\right)$ denotes the momentum (Lorentz index) of the vector field $V_{i}$ and for simplicity the spinors associated with the DM fields have been dropped. In terms of the sine $\left(s_{w}\right)$ and cosine $\left(c_{w}\right)$ of the weak mixing angle and the Wilson coefficients $C_{B}(\Lambda)$ and $C_{W}(\Lambda)$, the couplings $g_{V_{i} V_{j}}$ read

$$
\begin{aligned}
g_{A A} & =c_{w}^{2} C_{B}(\Lambda)+s_{w}^{2} C_{W}(\Lambda), \\
g_{A Z} & =-s_{w} c_{w}\left(C_{B}(\Lambda)-C_{W}(\Lambda)\right), \\
g_{Z Z} & =s_{w}^{2} C_{B}(\Lambda)+c_{w}^{2} C_{W}(\Lambda), \\
g_{W W} & =C_{W}(\Lambda) .
\end{aligned}
$$

These results do not coincide with the expressions reported in $[10,12,13]$. From $(16)$ we see that in the coupling $g_{A A}$ of DM to two photons, the Wilson coefficients $C_{B}(\Lambda)$ enter compared to $C_{W}(\Lambda)$ with a relative factor of $c_{w}^{2} / s_{w}^{2} \simeq 3.3$. On the other hand, in the case of the coupling between DM and $Z$-boson pairs $g_{Z Z}$, the dependence on $s_{w}$ and $c_{w}$ is reversed compared to $g_{A A}$. These properties explain why the limit on the new-physics scale $\Lambda$ from monophoton $\left[E_{T}+Z\left(\rightarrow \ell^{+} \ell^{-}\right), E_{T}+W / Z(\rightarrow\right.$ hadrons $)$, monojet and VBF $h \rightarrow$ invisible] searches is stronger (weaker) in the upper panel than in the lower panel of Fig. 2.

A second important feature worth noting is that channels with leptons in the final state typically lead to weaker restrictions on the parameter space than modes involving hadrons. This is a simple consequence of the fact that the electroweak SM gauge bosons dominantly decay hadronically. Numerically, one has $\operatorname{Br}\left(Z \rightarrow \ell^{+} \ell^{-}\right) \simeq 7 \%$ and $\operatorname{Br}\left(W \rightarrow \mu \nu_{\mu}\right) \simeq 11 \%$, while $\operatorname{Br}(Z \rightarrow$ hadrons $) \simeq 70 \%$ and $\operatorname{Br}(W \rightarrow$ hadrons $) \simeq 68 \%$ [36]. The strong suppression of the leptonic decay widths overcompensates the 
higher detection efficiencies of final states involving leptons, and as a result the LHC searches for $E_{T}+$ hadrons are superior to those looking for $E_{T}+$ leptons signals.

Our third observation is that the latest monojet data are evidently more constraining than the recent VBF $h \rightarrow$ invisible search. While these analyses explore the same final state, i.e. $E_{T}+2 j$, they probe quite different parts of the phase space. In fact, the selection criterion that has the biggest impact in our study is the rather loose missing transverse energy cut of $E_{T}>130 \mathrm{GeV}$ imposed in the VBF $h \rightarrow$ invisible search. This selection is tailored for a Higgs boson of $125 \mathrm{GeV}$, but fares less well if one tries to probe higher-dimensional operators of the form (2). Since the operators $O_{B}\left(O_{\tilde{B}}\right)$ and $O_{W}\left(O_{\tilde{W}}\right)$ produce a rather hard $E_{T}$ spectrum, more severe $E_{T}$ requirements allow for a cleaner separation between signal and SM background.

\section{B. Dependence on two Wilson coefficients}

Until now we have studied the constraints on the newphysics scale $\Lambda$ as a function of the DM mass $m_{\chi}$, keeping the values of the high-scale Wilson coefficients fixed. In the panels of Fig. 3 we instead show contours of constant $\Lambda$ in the $C_{B}(\Lambda)-C_{W}(\Lambda)$ plane. In all plots we employ $m_{\chi}=100 \mathrm{GeV}$ and set $C_{\tilde{B}}(\Lambda)=C_{\tilde{W}}(\Lambda)=0$. The first noticeable feature of the shown predictions is that only the monophoton signal depends more strongly on $C_{B}(\Lambda)$ than $C_{W}(\Lambda)$, while for all the other $E_{T}$ channels the situation is reversed. Second, with the exception of the monophoton case, one observes that the major axes of the elliptic contours in all panels are almost aligned with the $C_{W}(\Lambda)$ axes. This means that interference effects between contributions arising from $O_{B}$ and $O_{W}$ are small in all of these cases. The third important property following from the color shading of the depicted results is that currently either the newest monophoton or the monojet data provide the leading bounds in the entire $C_{B}(\Lambda)-C_{W}(\Lambda)$ plane. This feature is further illustrated by the upper panel in Fig. 4. In this plot the overlaid numbers indicate the search strategy that contributes the best sensitivity on $\Lambda$ at each point, with 1 and 5 corresponding to the monophoton and monojet channel, respectively. One sees that if the ratio of Wilson coefficients satisfies $\left|C_{B}(\Lambda) / C_{W}(\Lambda)\right| \gtrsim 1.5$ then the limit (4) gives rise to the strongest constraint, while in the remaining $C_{B}(\Lambda)-C_{W}(\Lambda)$ plane the bound (14) is most restrictive. The $\Lambda$ contours obtained by combining all available $E_{T}$ channels are depicted in the lower panel of Fig. 4.
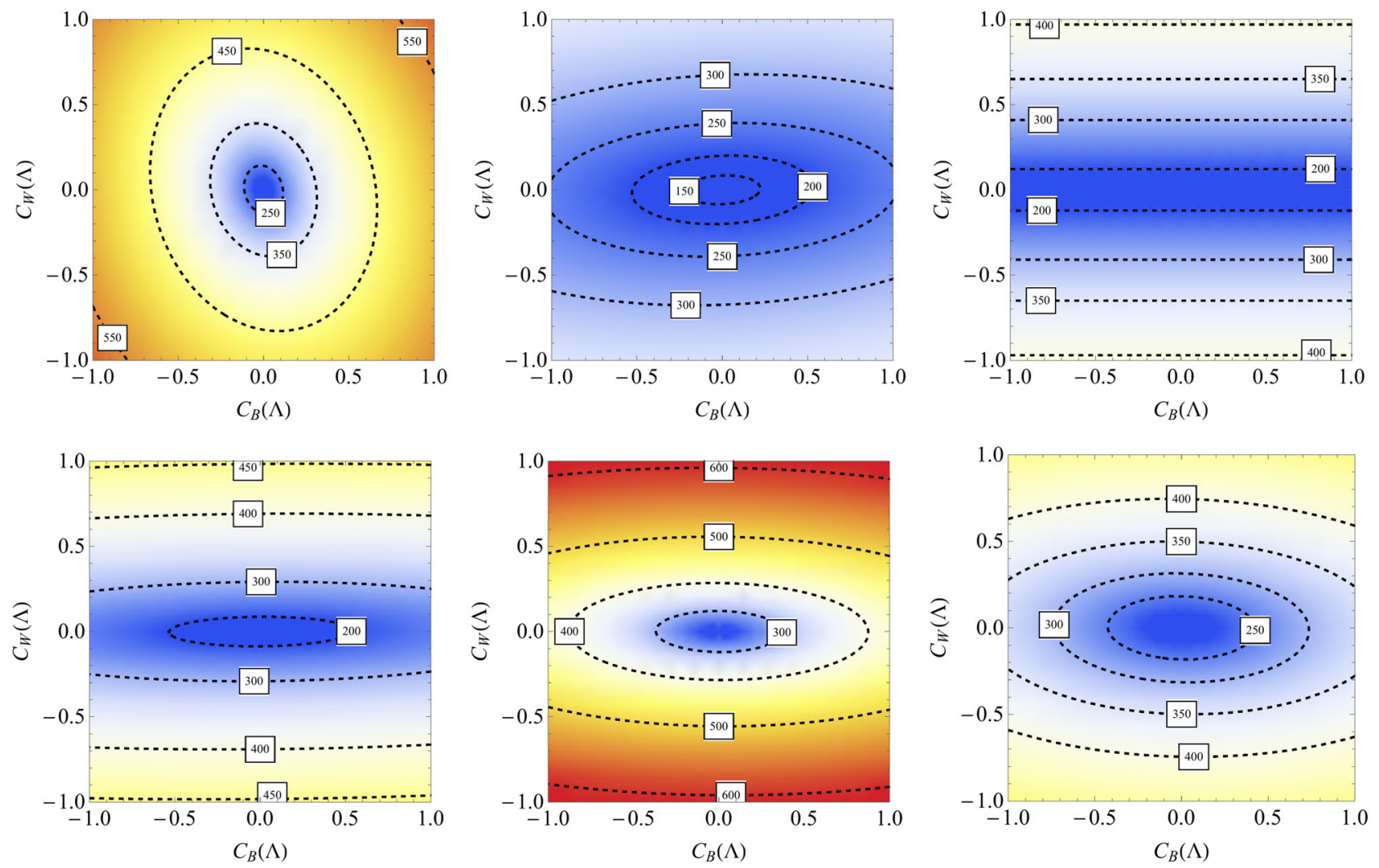

FIG. 3 (color online). Limits on $\Lambda$ in the $C_{B}(\Lambda)-C_{W}(\Lambda)$ plane. The different panels correspond to the monophoton (upper left), $E_{T}+Z\left(\rightarrow \ell^{+} \ell^{-}\right)$(upper middle), $E_{T}+W\left(\rightarrow \mu \nu_{\mu}\right)$ (upper right), $E_{T}+W / Z(\rightarrow$ hadrons) (lower left), monojet (lower middle) and VBF $h \rightarrow$ invisible (lower right) search. All results employ $m_{\chi}=100 \mathrm{GeV}$ and $C_{\tilde{B}}(\Lambda)=C_{\tilde{W}}(\Lambda)=0$. The contour labels indicate the value of the new-physics scale in units of GeV. 

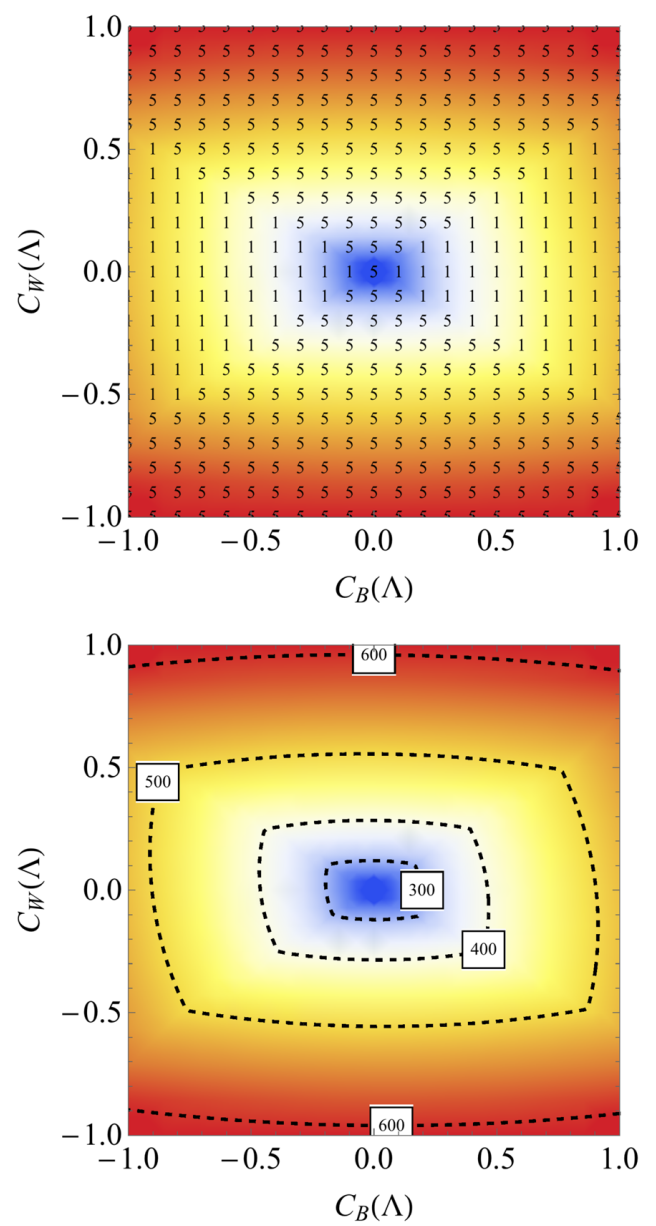

FIG. 4 (color online). Combination of the bounds on the new-physics scale in the $C_{B}(\Lambda)-C_{W}(\Lambda)$ plane, employing $m_{\chi}=100 \mathrm{GeV}$ and $C_{\tilde{B}}(\Lambda)=C_{\tilde{W}}(\Lambda)=0$. In the upper panel the search strategy that provides the leading constraint is indicated by the superimposed numbers, with 1 (5) representing the latest monophoton (monojet) search, while the lower panel shows the resulting contours of constant $\Lambda$ in units of $\mathrm{GeV}$.

Finally, we note that the values of $\Lambda$ that can be excluded with the current data are low compared to typical LHC energies. In order to go beyond the EFT description, one has to specify an ultraviolet (UV) completion, where the operators in (4) arise from a renormalizable theory after integrating out the heavy degrees of freedom mediating the interactions. UV-complete models that generate the operators $O_{B}$ and $O_{W}$ through loops of states charged under $U(1)_{Y}$ and/or $S U(2)_{L}$ have been proposed in [3] and their LHC signatures have been studied in [4]. If these new charged particles are light, the high- $p_{T}$ gauge bosons that participate in the $E_{T}$ processes considered here are able to resolve the substructure of the loops. This generically suppresses the cross sections compared to the EFT predictions [33], and thus will weaken the bounds on the interaction strengths of DM and the electroweak gauge bosons to some extent. Furthermore, the light charged mediators may be produced on-shell in $p p$ collisions, rendering direct LHC searches potentially more restrictive than $E_{T}$ searches. Making the above statements precise would require a study of a concrete UV completion.

\section{Future sensitivity}

It is also interesting to explore how the reach on the newphysics scale $\Lambda$ might improve at the $14 \mathrm{TeV}$ LHC. In what follows, we will only consider the monojet signal, applying the event selection criteria that have been used in the sensitivity study by ATLAS [37]. These read

$$
\begin{aligned}
p_{T, j_{1}} & >300 \mathrm{GeV}, & & \left|\eta_{j_{1}}\right|<2.0, \\
p_{T, j_{2}} & >50 \mathrm{GeV}, & & \left|\eta_{j_{2}}\right|<3.6, \\
\Delta \phi_{j E_{T}} & >0.5, & &
\end{aligned}
$$

and jets are reconstructed using an anti- $k_{t}$ algorithm with $R=0.4$. Events with a third jet of $p_{T, j_{3}}>50 \mathrm{GeV}$ and $\left|\eta_{j_{3}}\right|<3.6$ are vetoed and the missing transverse energy cut that we employ is $E_{T}>800 \mathrm{GeV}$. Note that compared to (11) the $p_{T, j_{1}}, p_{T, j_{2}}$ and $E_{T}$ thresholds are increased both to avoid pileup and to enhance the signal-over-background ratio. In order to determine the limits on the scale $\Lambda$, we take $\sigma_{\text {fid }}(p p \rightarrow Z(\rightarrow \bar{\nu} \nu)+j)=5.5 \mathrm{fb}$ [37], assuming a total systematic uncertainty on the SM background of $5 \%$. For the choice $C_{B}(\Lambda)=0, C_{W}(\Lambda)=1$ and $C_{\tilde{B}}(\Lambda)=$ $C_{\tilde{W}}(\Lambda)=0$, we find that with $25 \mathrm{fb}^{-1}$ of data, corresponding to the first year of running after the LHC upgrade to $14 \mathrm{TeV}$, one may be able to set a 95\% C.L. bound of $\Lambda \gtrsim 1.3 \mathrm{TeV}$ for $m_{\chi} \lesssim 100 \mathrm{GeV}$. Compared to the present limit, this corresponds to an improvement of the bound on $\Lambda$ by more than a factor of 2 . With $300 \mathrm{fb}^{-1}$ and $3000 \mathrm{fb}^{-1}$ of accumulated data, we obtain instead $\Lambda \gtrsim 1.4 \mathrm{TeV}$. These numbers make clear that at $14 \mathrm{TeV}$ the sensitivity of $E_{T}+j$ searches will rather soon be limited by systematic uncertainties associated to the irreducible SM background. Finding to what extent this limitation can be evaded by an optimization of the monojet searches and/or an improved understanding of the $p p \rightarrow Z(\rightarrow \bar{\nu} \nu)+j$ channel would require a dedicated study. Such an analysis is beyond the scope of this work.

\section{Analysis of jet-jet angular correlations}

So far we have analyzed only observables that are insensitive to whether the $E_{T}$ signal is generated by an insertion of the effective operator $O_{B}\left(O_{W}\right)$ or $O_{\tilde{B}}\left(O_{\tilde{W}}\right)$. This ambiguity can however be resolved by measuring the azimuthal angle difference $\Delta \phi_{j_{1} j_{2}}$ of forward jets produced in $E_{T}+2 j$ events [9,21]. Besides the cuts (17), we impose the following VBF-like selection requirements in our analysis

$\eta_{j_{1}} \cdot \eta_{j_{2}}<0, \quad \Delta \eta_{j_{1} j_{2}}>2, \quad m_{j_{1} j_{2}}>1100 \mathrm{GeV}$ 
Here the cut on the pseudorapidity separation helps to sculpt the angular correlations between the tagging jets, while the dijet invariant mass threshold improves the signal-over-background ratio.

In order to understand why the operators $O_{B}\left(O_{W}\right)$ and $O_{\tilde{B}}\left(O_{\tilde{W}}\right)$ lead to different jet-jet angular correlations, one has to consider their Feynman rules. In the case of the operators containing regular field strength tensors this has already been done in (15), while for their dual counterparts we obtain

$$
\frac{2 i}{\Lambda^{3}} g_{V_{1} V_{2}} \epsilon^{\mu_{1} \mu_{2} \nu \lambda}\left(p_{1 \nu} p_{2 \lambda}-p_{1 \lambda} p_{2 \nu}\right),
$$

with $g_{V_{i} V_{j}}$ given in (16). The selection cuts (18) emphasize the parts of the phase space where the external partons experience only a small energy loss and the momentum components of the tagging jets in the beam direction are much greater than those in the transverse plane. In this limit the structure of the $p p \rightarrow E_{T}+2 j$ matrix elements is straightforward to work out [38]. In the case of the effective operator $O_{W}$, one gets for instance $\mathcal{M}_{W} \sim$ $J_{1}^{\mu_{1}} J_{2}^{\mu_{2}}\left(g_{\mu_{1} \mu_{2}} p_{1} \cdot p_{2}-p_{1 \mu_{1}} p_{2 \mu_{2}}\right) \sim \vec{p}_{T, j_{1}} \cdot \vec{p}_{T, j_{2}}$, while for $O_{\tilde{W}}$ one arrives instead at $\mathcal{M}_{\tilde{W}} \sim \epsilon_{\mu_{1} \mu_{2} \nu \lambda} J_{1}^{\mu_{1}} J_{2}^{\mu_{2}} p_{1}^{\nu} p_{2}^{\lambda} \sim$ $\vec{p}_{T, j_{1}} \times \vec{p}_{T, j_{2}}$. Here $J_{i}$ and $p_{i}$ denote the currents and momenta of the electroweak gauge bosons that partake in the scattering. These simple arguments imply that the $\Delta \phi_{j_{1} j_{2}}$ spectrum corresponding to $O_{W}$ should be enhanced for collinear tagging jets, $\Delta \phi_{j_{1} j_{2}}=0$, while for $\Delta \phi_{j_{1} j_{2}}=$ $\pi / 2$ it should show an approximate zero. In the case of $O_{\tilde{W}}$, on the other hand, the $\Delta \phi_{j_{1} j_{2}}$ distribution should have a dip if the two jets are collinear, $\Delta \phi_{j_{1} j_{2}}=0$, or back-to-back, $\Delta \phi_{j_{1} j_{2}}=\pi$. Note that the above arguments do not depend on the chirality of the DM current. This means that $O_{B}, O_{W}$, $O_{\tilde{B}}, O_{\tilde{W}}$ and the operators obtained from (2) by replacing $\bar{\chi} \chi$ with $\bar{\chi} \gamma_{5} \chi$ lead to very similar jet-jet angular correlations, as we have explicitly verified.

In Fig. 5 we plot the $\Delta \phi_{j_{1} j_{2}}$ spectra for the choices $C_{B}(\Lambda)=0, \quad C_{W}(\Lambda)=1$ (red curve) and $C_{\tilde{B}}(\Lambda)=0$, $C_{\tilde{W}}(\Lambda)=1$ (blue curve). All shown predictions are obtained for the $14 \mathrm{TeV}$ LHC and employ $\Lambda=1 \mathrm{TeV}$ and $m_{\chi}=100 \mathrm{GeV}$. The fiducial signal cross sections amount to $1.0 \mathrm{fb}$, independently of whether the insertion of $O_{W}$ or $O_{\tilde{W}}$ is considered. The expected sinelike (cosinelike) behavior of the modulation in the azimuthal angle distribution corresponding to $O_{W}\left(O_{\tilde{W}}\right)$ is clearly visible in the figure. These shapes should be contrasted with the spectrum of the dominant SM background process $p p \rightarrow$ $Z(\rightarrow \bar{\nu} \nu)+2 j$ (black curve), which is rather flat for values $\Delta \phi_{j_{1} j_{2}} \lesssim 2.6$ and then rapidly drops to zero. The corresponding fiducial cross section is $0.35 \mathrm{fb}$, implying a signal-over-background ratio of $S / \sqrt{B} \simeq 8.4,29$ and 93 for $25 \mathrm{fb}^{-1}, 300 \mathrm{fb}^{-1}$ and $3000 \mathrm{fb}^{-1}$ of data, respectively.

The given $S / \sqrt{B}$ values imply that running the LHC for a couple of years at $14 \mathrm{TeV}$ should provide a

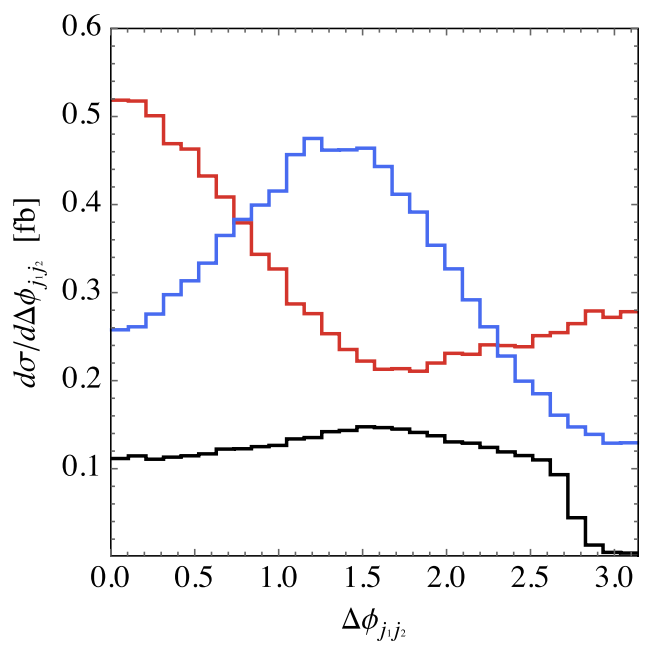

FIG. 5 (color online). Azimuthal angle distributions at the $14 \mathrm{TeV}$ LHC. The signal curves correspond to $C_{B}(\Lambda)=0$, $C_{W}(\Lambda)=1$ (red) and $C_{\tilde{B}}(\Lambda)=0, C_{\tilde{W}}(\Lambda)=1$ (blue), and both use $\Lambda=1 \mathrm{TeV}$ and $m_{\chi}=100 \mathrm{GeV}$. For comparison the prediction of the dominant SM background process $p p \rightarrow$ $Z(\rightarrow \bar{\nu} \nu)+2 j$ (black) employing the same event selection criteria is shown as well.

sufficient number of events to analyze the jet-jet angular correlations. To quantify this statement, we use a toy MC and generate event samples for both signals and background corresponding to $300 \mathrm{fb}^{-1}$ and $3000 \mathrm{fb}^{-1}$ of luminosity. The resulting differential cross sections are then fitted to [39]

$$
\frac{1}{\sigma} \frac{d \sigma}{d \Delta \phi_{j_{1} j_{2}}}=\sum_{n=0}^{2} a_{n} \cos \left(n \Delta \phi_{j_{1} j_{2}}\right) .
$$

The coefficient $a_{0}$ is fixed by the normalization of the $\Delta \phi_{j_{1} j_{2}}$ spectrum, and the ratio $r_{1}=a_{1} / a_{0}$ turns out to be rather insensitive to which type of higher-dimensional interactions is considered. In contrast, the combination $r_{2}=a_{2} / a_{0}$ is a measure of the $C P$ nature of the interactions that lead to the $2 j$ final state (see e.g. $[38,39]$ ). This ratio is expected to be positive (negative) for an insertion of $O_{B}\left(O_{\tilde{B}}\right)$ and $O_{W}\left(O_{\tilde{W}}\right)$. We stress that by considering normalized $\Delta \phi_{j_{1} j_{2}}$ distributions, theoretical uncertainties are reduced and that the predictions become fairly independent of EFT assumptions [21].

In Fig. 6 we present the results of our toy MC. The upper panel (lower panel) corresponds to $300 \mathrm{fb}^{-1}\left(3000 \mathrm{fb}^{-1}\right)$ of LHC data collected at $14 \mathrm{TeV}$. The expected azimuthal angle distributions for the signal plus background predictions are colored blue (red) for $O_{W}\left(O_{\tilde{W}}\right)$. For comparison, the SM-only result (grey) divided by a factor of 3 is also shown. The solid curves illustrate the best fits to (20), restricting the rapidity separation $\Delta \phi_{j_{1} j_{2}}$ to the range $[0,2.5]$. For $300 \mathrm{fb}^{-1}$ of data, we obtain for $r_{2}$ the central values and uncertainties 

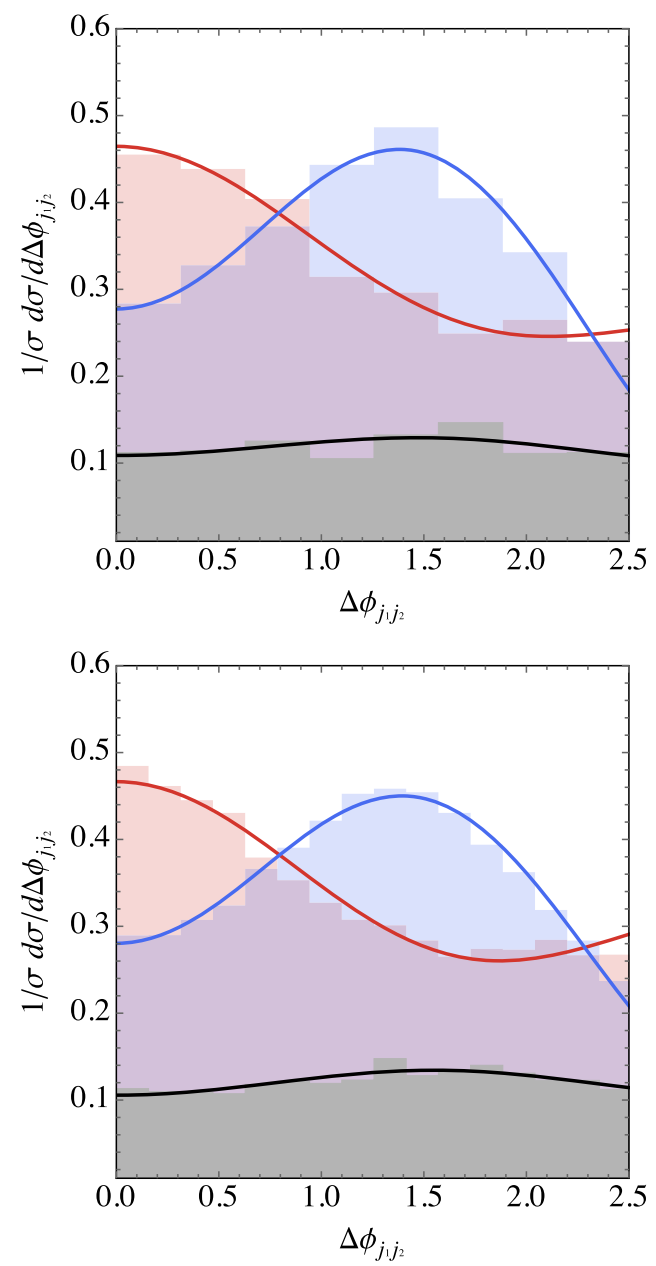

FIG. 6 (color online). Normalized $\Delta \phi_{j_{1} j_{2}}$ distributions for $300 \mathrm{fb}^{-1}$ (upper panel) and $3000 \mathrm{fb}^{-1}$ (lower panel) of $14 \mathrm{TeV}$ LHC data. The red (blue) histogram shows the signal plus background prediction for $O_{W}\left(O_{\tilde{W}}\right)$. The grey bar chart represents the expected SM background, which for better visibility, has been rescaled by a factor of $1 / 3$. The solid curves indicate the best fits of the form $a_{0}+a_{1} \cos \Delta \phi_{j_{1} j_{2}}+a_{2} \cos \left(2 \Delta \phi_{j_{1} j_{2}}\right)$. See text for additional explanations.

$$
\begin{aligned}
\left(r_{2}\right)_{W+\mathrm{SM}} & =0.15 \pm 0.10, \\
\left(r_{2}\right)_{\tilde{W}+\mathrm{SM}} & =-0.45 \pm 0.14, \\
\left(r_{2}\right)_{\mathrm{SM}} & =-0.12 \pm 0.22 .
\end{aligned}
$$

In the case of $3000 \mathrm{fb}^{-1}$ of luminosity, we find instead

$$
\begin{aligned}
\left(r_{2}\right)_{W+\mathrm{SM}} & =0.18 \pm 0.03 \\
\left(r_{2}\right)_{\tilde{W}+\mathrm{SM}} & =-0.40 \pm 0.04, \\
\left(r_{2}\right)_{\mathrm{SM}} & =-0.13 \pm 0.07 .
\end{aligned}
$$

We observe that for $O_{W}\left(O_{\tilde{W}}\right)$ the combination $r_{2}$ is indeed positive (negative). Defining a significance as $s_{k}=$ $\left(\left(r_{2}\right)_{k+\mathrm{SM}}-\left(r_{2}\right)_{\mathrm{SM}}\right) /\left(\Delta r_{2}\right)_{k+\mathrm{SM}}$, we get from (21) the values $s_{W}=2.7$ and $s_{\tilde{W}}=-2.4$, while (22) leads to $s_{W}=$ 10.3 and $s_{\tilde{W}}=-6.8$. Our toy MC study corresponding to $300 \mathrm{fb}^{-1}\left(3000 \mathrm{fb}^{-1}\right)$ of data hence suggests that a distinction between the azimuthal angle distributions of $O_{W}$ and $O_{\tilde{W}}$ at the $5 \sigma(17 \sigma)$ level should be possible at the $14 \mathrm{TeV}$ LHC. We emphasize that our toy study assumes a perfect detector and that we have not optimized the cuts (18) to achieve the best significance. Once the data are on tape, it will become an experimental issue of how stringent the VBF-like selections can be made to extract the most information on the jet-jet angular correlations for a given limited sample size.

\section{CONCLUSIONS}

In this article we have studied LHC constraints on effective dimension-7 operators that couple DM to the SM electroweak gauge bosons and emphasized the complementarity of different $E_{T}$ searches for constraining the associated Wilson coefficients. Focusing on the interactions that induce only velocity-suppressed annihilation rates, we have combined the information on all individual search modes that are available after LHC run-1. In this way we are able to derive bounds on the new-physics scale $\Lambda$ that exceed all previous limits. Our studies show that at present, depending on the choice of parameters, either monophoton or monojet searches provide the most severe constraints on the considered dimension-7 interactions. For DM masses $m_{\chi} \lesssim 100 \mathrm{GeV}$ and Wilson coefficients $\left|C_{k}(\Lambda)\right| \simeq 1$, the existing $8 \mathrm{TeV}$ LHC searches allow us to exclude values of $\Lambda$ below about $600 \mathrm{GeV}$ at 95\% C.L. The improved reach of $E_{T}$ analyses in 2015 and beyond is also studied, finding that with $25 \mathrm{fb}^{-1}$ of $14 \mathrm{TeV}$ data, LHC monojet searches should be able to improve the latter bound to approximately $1.3 \mathrm{TeV}$. Beyond this point further progress will be hindered by the imperfect understanding of irreducible SM backgrounds such as $p p \rightarrow Z(\rightarrow \bar{\nu} \nu)+j$. Finding ways to overcome these limitations will be crucial to exploit the full physics potential of $E_{T}$ searches to be carried out at later stages of the LHC.

We have furthermore emphasized that given the large statistics expected at the phase- 1 and phase- 2 upgrades of the $14 \mathrm{TeV}$ LHC, $E_{T}$ searches should be able to not only determine integrated, but also differential cross sections. From the theoretical point of view, such normalized distributions have the clear advantage that compared to the total cross sections theoretical uncertainties are reduced and that the obtained predictions depend only weakly on the assumptions underlying the EFT description. As an example we have explored the prospects to measure jet-jet angular correlations in $E_{T}+2 j$ events. Taking into account the pseudorapidity correlations of the two tagging jets, the resulting distributions in the azimuthal angle separation $\Delta \phi_{j_{1} j_{2}}$ exhibit the relative strength of $C P$-even and $C P$-odd interactions of DM with gauge boson pairs. Our toy MC 
studies indicate that already with $300 \mathrm{fb}^{-1}$ of data a distinction between the new-physics and the SM-only hypotheses can be achieved at a statistically significant level, and that the sensitivity of the discussed searches is greatly improved by going to $3000 \mathrm{fb}^{-1}$ of luminosity. A more precise determination of the analyzing power, including systematic uncertainties, would require a full detector simulation, which is beyond the scope of the present article. We however believe that it is imperative that the ATLAS and CMS collaborations direct some activity towards the study of differential distributions of final states like $E_{T}+2 j$.

\section{ACKNOWLEDGMENTS}

We thank Tim Tait for clarifying discussions concerning his work [10] and are grateful to Benjamin Fuks, Emanuele Re and Giulia Zanderighi for help with MadGraph and/or PYTHIA. A. C. is supported by a Marie Curie Intra-European Fellowship of the European Community's 7th Framework Programme under Contract No. PIEF-GA-2012-326948. U.H. acknowledges the hospitality and support of the CERN theory division and the Munich Institute for Astroand Particle Physics (MIAPP) of the DFG cluster of excellence "Origin and Structure of the Universe." The research of A. H. is supported by a STFC Postgraduate Studentship.
[1] A. Askew, S. Chauhan, B. Penning, W. Shepherd, and M. Tripathi, Searching for dark matter at hadron colliders, Int. J. Mod. Phys. A 29, 1430041 (2014).

[2] N. Weiner and I. Yavin, How dark are majorana WIMPs? Signals from MiDM and Rayleigh dark matter, Phys. Rev. D 86, 075021 (2012).

[3] N. Weiner and I. Yavin, UV completions of magnetic inelastic dark matter and RayDM for the Fermi line(s), Phys. Rev. D 87, 023523 (2013).

[4] J. Liu, B. Shuve, N. Weiner, and I. Yavin, Looking for new charged states at the LHC: Signatures of magnetic and Rayleigh dark matter, J. High Energy Phys. 07 (2013) 144.

[5] A. Rajaraman, T. M. P. Tait, and D. Whiteson, Two lines or not two lines? That is the question of gamma ray spectra, J. Cosmol. Astropart. Phys. 09 (2012) 003.

[6] M. T. Frandsen, U. Haisch, F. Kahlhoefer, P. Mertsch, and K. Schmidt-Hoberg, Loop-induced dark matter direct detection signals from gamma-ray lines, J. Cosmol. Astropart. Phys. 10 (2012) 033.

[7] A. Rajaraman, T. M. P. Tait, and A. M. Wijangco, Effective theories of gamma-ray lines from dark matter annihilation, Phys. Dark Univ. 2, 17 (2013).

[8] A. Crivellin and U. Haisch, Dark matter direct detection constraints from gauge bosons loops, Phys. Rev. D 90, 115011 (2014).

[9] R. C. Cotta, J. L. Hewett, M. P. Le, and T. G. Rizzo, Bounds on dark matter interactions with electroweak gauge bosons, Phys. Rev. D 88, 116009 (2013).

[10] L. M. Carpenter, A. Nelson, C. Shimmin, T. M. P. Tait, and D. Whiteson, Collider searches for dark matter in events with a $\mathrm{Z}$ boson and missing energy, Phys. Rev. D 87, 074005 (2013).

[11] G. Aad et al. (ATLAS Collaboration), Search for dark matter in events with a $\mathrm{Z}$ boson and missing transverse momentum in pp collisions at $\sqrt{s}=8 \mathrm{TeV}$ with the ATLAS detector, Phys. Rev. D 90, 012004 (2014).

[12] A. Nelson, L. M. Carpenter, R. Cotta, A. Johnstone, and D. Whiteson, Confronting the Fermi line with LHC data: An effective theory of dark matter interaction with photons, Phys. Rev. D 89, 056011 (2014).
[13] N. Lopez, L. M. Carpenter, R. Cotta, M. Frate, N. Zhou, and D. Whiteson, Collider bounds on indirect dark matter searches: The $W W$ final state, Phys. Rev. D 89, 115013 (2014).

[14] V. Khachatryan et al. (CMS Collaboration), Search for new phenomena in monophoton final states in proton-proton collisions at $\sqrt{s}=8 \mathrm{TeV}$, arXiv: 1410.8812 .

[15] G. Aad et al. (ATLAS Collaboration), Search for new phenomena in events with a photon and missing transverse momentum in $p p$ collisions at $\sqrt{s}=8 \mathrm{TeV}$ with the ATLAS detector, Phys. Rev. D 91, 012008 (2015).

[16] G. Aad et al. (ATLAS Collaboration), Search for Dark Matter in Events with a Hadronically Decaying $\mathrm{W}$ or $\mathrm{Z}$ Boson and Missing Transverse Momentum in $p p$ Collisions at $\sqrt{s}=8 \mathrm{TeV}$ with the ATLAS Detector, Phys. Rev. Lett. 112, 041802 (2014).

[17] S. Chatrchyan et al. (CMS Collaboration), Search for invisible decays of Higgs bosons in the vector boson fusion and associated $\mathrm{ZH}$ production modes, Eur. Phys. J. C 74, 2980 (2014).

[18] G. Aad et al. (ATLAS Collaboration), Search for new particles in events with one lepton and missing transverse momentum in $p p$ collisions at $\sqrt{s}=8 \mathrm{TeV}$ with the ATLAS detector, J. High Energy Phys. 09 (2014) 037.

[19] V. Khachatryan et al. (CMS Collaboration), Search for physics beyond the standard model in final states with a lepton and missing transverse energy in proton-proton collisions at $\sqrt{s}=8 \mathrm{TeV}$, arXiv:1408.2745.

[20] V. Khachatryan et al. (CMS Collaboration), Search for dark matter, extra dimensions, and unparticles in monojet events in proton-proton collisions at $\sqrt{s}=8 \mathrm{TeV}$, arXiv:1408.3583.

[21] U. Haisch, A. Hibbs, and E. Re, Determining the structure of dark-matter couplings at the LHC, Phys. Rev. D 89, 034009 (2014).

[22] Y. L. Dokshitzer, G. D. Leder, S. Moretti, and B. R. Webber, Better jet clustering algorithms, J. High Energy Phys. 08 (1997) 001.

[23] J. M. Butterworth, A. R. Davison, M. Rubin, and G. P. Salam, Jet Substructure as a New Higgs Search Channel at the LHC, Phys. Rev. Lett. 100, 242001 (2008). 
[24] M. Cacciari, G. P. Salam, and G. Soyez, The anti- $k(t)$ jet clustering algorithm, J. High Energy Phys. 04 (2008) 063.

[25] U. Haisch, F. Kahlhoefer, and E. Re, QCD effects in monojet searches for dark matter, J. High Energy Phys. 12 (2013) 007.

[26] N. D. Christensen and C. Duhr, FeynRules - Feynman rules made easy, Comput. Phys. Commun. 180, 1614 (2009).

[27] C. Degrande, C. Duhr, B. Fuks, D. Grellscheid, O. Mattelaer, and T. Reiter, UFO - The Universal FeynRules Output, Comput. Phys. Commun. 183, 1201 (2012).

[28] J. Alwall, M. Herquet, F. Maltoni, O. Mattelaer, and T. Stelzer, MadGraph 5: Going beyond, J. High Energy Phys. 06 (2011) 128.

[29] J. Pumplin, D. R. Stump, J. Huston, H. L. Lai, P. M. Nadolsky, and W. K. Tung, New generation of parton distributions with uncertainties from global QCD analysis, J. High Energy Phys. 07 (2002) 012.

[30] T. Sjostrand, S. Mrenna, and P.Z. Skands, A brief introduction to PYTHIA 8.1, Comput. Phys. Commun. 178, 852 (2008).

[31] M. Cacciari, G. P. Salam, and G. Soyez, FastJet user manual, Eur. Phys. J. C 72, 1896 (2012).
[32] J. de Favereau, C. Delaere, P. Demin, A. Giammanco, V. Lemaître, A. Mertens, and M. Selvaggi (DELPHES 3 Collaboration), DELPHES 3, A modular framework for fast simulation of a generic collider experiment, J. High Energy Phys. 02 (2014) 057.

[33] U. Haisch, F. Kahlhoefer, and J. Unwin, The impact of heavy-quark loops on LHC dark matter searches, J. High Energy Phys. 07 (2013) 125.

[34] U. Haisch and F. Kahlhoefer, On the importance of loopinduced spin-independent interactions for dark matter direct detection, J. Cosmol. Astropart. Phys. 04 (2013) 050.

[35] U. Haisch and E. Re, Simplified dark matter top-quark interactions at the LHC, arXiv:1503.00691.

[36] K. A. Olive et al. (Particle Data Group Collaboration), Review of particle physics, Chin. Phys. C 38, 090001 (2014).

[37] ATLAS Collaboration, http://cds.cern.ch/record/1708859/ files/ATL-COM-PHYS-2014-549.pdf.

[38] T. Plehn, D. L. Rainwater, and D. Zeppenfeld, Determining the Structure of Higgs Couplings at the LHC, Phys. Rev. Lett. 88, 051801 (2002).

[39] V. Hankele, G. Klamke, and D. Zeppenfeld, Higgs +2 jets as a probe for $C P$ properties, arXiv:hep-ph/0605117. 